\title{
VERTEBRATES AND EVOLUTION ${ }^{1}$
}

\author{
Philip D. Gingerich \\ Museum of Paleontology, The University of Michigan, Ann Arbor, Michigan 48109-1079
}

Received November 6, 1997.

\begin{abstract}
Patterns and Processes of Vertebrate Evolution is a masterful overview of evolution as it is understood by many vertebrate paleontologists and others today. The author is best known for beautifully illustrated descriptions and synthetic comparative studies of Paleozoic amphibians and, more recently, marine reptiles. He had the courage to revise Alfred Sherwood Romer's classic Vertebrate Paleontology text and reference book, and here attempts an update of George Gaylord Simpson's Major Features of Evolution. Carroll writes clearly and rapidly-the whole is remarkably up to date, with meaningful incorporation of much current literature. The book exhibits Carroll's usual flair for illustration. All in all this is a fine book.
\end{abstract}

At the same time, I have some reservations. Patterns and Processes of Vertebrate Evolution seems to me to be too masterful an overview of the state of paleontological thought on evolution at a time when this field needs critical dissection rather than overview: more numbers and fewer words. The book is inconsistent, even contradictory, in its major themes. Carroll claims in the preface (p. xii) that his conclusions "differ from those of Darwin, the modern evolutionary synthesis, and the theory of punctuated equilibrium in being much more pluralistic. The rates and patterns of evolution as well as the forces of evolutionary change are observed to be extremely diverse and variable, differing significantly between modern populations and species and those illustrated by the fossil record over millions and hundreds of millions of years."

Pluralism can be a virtue, but it is also a codeword for indecisiveness. In my experience nothing about rates or patterns in the fossil record differs "significantly" from what we see today when differences in scale and sampling are taken into account (see below). Carroll comes to this conclusion too, more or less, in the end. The book confused me in places and as a text I think it will confuse students (polling my students, some clearly like it more than others).

It is helpful to distinguish patterns and processes, as Carroll and many others do. I would go further. No one can understand evolution as a process without making some kind of conceptual or, better, computer model of it. Paleontologists generally shun modeling, sometimes on the theory that the evolution of life is a unique event that simply cannot be modeled. This is true of the pattern as a whole through Phanerozoic time, but the generational process is simpler and operates over and over again on a very different generation-togeneration time scale. Lineages have changed and branched millions of times in the history of life. We don't know exactly

\footnotetext{
${ }^{1}$ Patterns and Processes of Vertebrate Evolution. Robert L. Carroll. 1997. Cambridge University Press, Cambridge, U.K., xiii + 448 pp. HB $\$ 70.00$, ISBN 0-521-47232-6; and PB $\$ 24.95,0-521$ 47809-X.
}

how much any one lineage has changed, nor how many times all have branched, but we can estimate these, and the details matter less than the general model. Modeling is the only way to learn what patterns a process produces.

Darwin's theory of the evolutionary process, expressed in his figure in the Origin (and reproduced by Carroll as Figure 1.1) uses a bivariate graph of morphology (unscaled) versus time (on a scale of about $10^{3}$ generations) to illustrate that: (1) evolution is gradual; (2) it is slow enough to see; (3) change in a lineage is normally distributed in each time unit (with some unspecified standard deviation) about a mean or expectation of zero change (stasis); (4) lineages branch every $10^{3}$ generations or so; and (5) some proportion go extinct in every multigeneration interval, due in part to competitive interaction.

Thanks to computers, this is easy to program and literally see graphically. Our graphs don't look exactly like Darwin's because his diagram was drawn by hand with a lot of builtin regularity. I say our "graphs" because one reason to program Darwin's process is to see the variety of patterns a simple program will produce: a random walk divides and becomes two, and over time branches flourish, merely survive, or go extinct. As David Raup and others have shown, one cannot help but be impressed that such simulated patterns mimic much of what we see in the fossil record (modeling is not discussed by Carroll).

Darwin's model and our computer simulations are important here because in the very first chapter (pp. 2-3) Carroll as paleontologist claims that evolutionary patterns in the major groups of "vascular plants, vertebrates, and nonvertebrate metazoans" through much of Phanerozoic time are conspicuously different from Darwinian expectation because: (1) they have relatively few major lineages (groups), all of which are very distinct from one another; (2) they have gaps between the lineages that indicate adaptive space was not fully occupied; (3) major lineages appear suddenly in the fossil record instead of showing gradual and continuous change through time-exhibiting when they appear many of the features by which their modern representatives are recognized; (4) as a consequence, evolution occurs much more rapidly between groups than within groups; (5) for most of their evolutionary history, fundamental aspects of the anatomy and way of life of these major (groups) do not change significantly; finally (6) very few intermediates between groups are known in the fossil record. Carroll (pp. 88-90, 106, 110, 145) criticizes Niles Eldredge, Stephen Gould, and Steven Stanley's theory of punctuated equilibria at the level of species and genera, but comes across as an ardent punctuationist for evolution above this level.

Now, returning to our Darwinian computer model, let each simulation run for a long time, degrade each record to rep- 
resent incomplete sampling (introducing a host of potential misperceptions), and classify lineages in hindsight into "relatively few" major groups based on morphological similarity through time. What do we have? Pretty much what we think we see in the fossil record (Carroll's points 1-6 in the previous paragraph). Patterns of change in a few lineages over short intervals of time (Darwin's figure and Carroll's fig. 1.1) look different from patterns of change in major groups over very long intervals of time (Carroll's figs. 1.2-1.4 and our simulation results) when we know both were generated by the same process. The same process looks different when sampled on different scales of time. Time scale is important. I wouldn't use this book to teach about evolution without making students program Darwin's model and compare the results on different time scales.

The importance of time scale comes up again in Chapters 8 through 11 dealing with evolutionary constraints. One of the counter-intuitive things about random walks is that they never go very far from where they start, no matter how much time goes by. Evolution is fundamentally a random process in the sense that the variation on which selection acts is generated this way. Weak selection allows lineages to behave like random walks, as they do in our computer model. The limited range of random walks is not one of the many evolutionary constraints mentioned by Carroll, but since these do stay close to home it is a constraint that must be considered. Think of it as a contingent dependency on evolution's starting point. Here again, comparison of the long and shortterm patterns or traces of a random walk shows these to look very different, while the process or algorithm that generated both is, of course, one and the same.

Carroll claims that rates of evolution in organisms living today and those living in the geological past are "extremely diverse and variable." Yet when he reviews Peter and Rosemary Grant's study of modern Galapagos finches (pp. 3852), he reports Geospiza fortis born in 1978 to have been about 0.3 standard deviations larger than those born in 1976. Reviewing my study of 50 million-year-old dawn horses ( $p$. 76), Carroll reports Hyracotherium grangeri to have changed at a base rate of about 0.2 standard deviations per generation. These rates, both on a generational time scale, are not "extremely diverse" - they are virtually the same (in different organisms, studied on different time scales, at different times in earth history)! Base rates on the order of $0.1-0.2$ standard deviations per generation come up again and again in studies of evolutionary rates in the laboratory, the field, and the fossil record.

The influence of systems of classification on concepts of evolutionary patterns is reviewed in Chapter 7. Here Carroll returns to Darwin's Origin illustration, claiming (p. 145) that it "is matched in many respects by Greenwood's diagram of the evolution of cichlids in Lake Victoria" (Fig. 7.1)-I wish Carroll would explain how. Both have branches and both are ambiguously scaled, but that is about the only resemblance I see. I also don't like dismissal of Linnean systematics as "typological" (p. 149) because "species, whether living or fossil, are based on type specimens." As a student, I read enough Simpson to know that type specimens are mere reference points in variable samples, with no implication of typology (though type and typology do of course share a common etymology). Much of this chapter is on Hennigian systematics, monophyly (holophyly) and paraphyly, etc. I have no problem with Hennigian systematics in theory: derived characters (p. 150) are wonderful-once you know they are derived. Carroll's point about Condylarthra (pp. 152-153) escapes me: why should paraphyly make me write 'Protungulatum + Triisodontine mesconychids + Hyopsodontidae + Periptychidae + Minchinella + Radinskya' in place of 'Condylarthra' when, seemingly and hopefully, all of these constituents gave rise to descendant radiations too?

I mentioned constraints above, and Chapters 8-11, onethird of the book, are on evolutionary, genetic, developmental, and physical constraints on patterns and processes. These chapters are a strength of the book. Chapter 12 on major evolutionary transitions focuses on the origin of terrestrial amphibians from aquatic fish (7 pp.), the origin of birds from theropod dinosaurs (18 pp.), the origin of aquatic mosasaurs from terrestrial lizards (6 pp.), and the origin of aquatic whales from terrestrial mesonychids ( 7 pp.). Carroll draws the following general conclusions from these case studies: (1) major transitions span from one to 15-20 million years of geological time; (2) rates of change may have been high, but more importantly the direction of change was nearly constant (this is not demonstrated); (3) transitions often involved a mosaic shifting balance of old and new adaptations; (4) evolution of the flight apparatus in birds occurred more rapidly in proximal than distal elements of the forelimb; $(5)$ the transition from theropods to Archaeopteryx involved less change than that from Archaeopteryx to later birds; (6) there is no reason to think that species-level change took place any differently in these transitions than it does in general; and (7) major transitions are often contingent on factors that are impossible to predict from living species.

Chapters 13 and 14 are on patterns of diversification, and "forces of evolution" associated with these. Carroll concludes that the fossil record demonstrates the history of life to be "punctuated" (his word, p. 360 ) by a relatively small number of rapid radiations that resulted in the appearance of a wide range of anatomical patterns and adaptive modes (again, this is a view in hindsight). These radiations were concentrated in intervals of 10 million years or less, and often occurred in environments almost totally lacking in competitors or predators. Other radiations took place more slowly in environments already occupied by related forms. The largest-scale and most rapid radiations can be viewed as responses to mass extinctions, changing configurations of continents, modifications of genetic and developmental processes, and increasing levels of atmospheric oxygen.

Finally, in Chapter 15, Carroll asks whether a distinct theory of macroevolution is necessary. He writes (p. 391) "One of the outstanding problems in large-scale evolution has been the origin of major taxa, such as tetrapods, birds, and whales, that had appeared to arise suddenly, without any obvious ancestors, over a comparatively short period of time. Increased knowledge of the fossil record has greatly increased our understanding of these and other transitions, and shown that they do not necessarily require processes that differ from those known to occur at much lower taxonomic levels." He concludes (p. 392) that "Although formulation of a distinct theory of macroevolution does not 
appear to be justified, it may be convenient to retain the terms microevolution and macroevolution to describe the different patterns of evolution that are observed as the level of populations and species versus higher taxonomic levels and time spans exceeding 5-10 million years." Where is the bluster about pluralism superseding Darwin and superseding the modern synthesis that we read in the preface?
We learn more about evolution the more we study it, but Darwin and Simpson and others (including Carroll in his studies of amphibians and marine reptiles) have given us a solid foundation to build on. I sense that the beginning of this book would have been different if Carroll had known at the start what he knew by the end.

Book Review Editor: J. Coyne

\title{
A TOUGH CELL'
}

\author{
GREGORY A. WRAY \\ Department of Ecology and Evolution, State University of New York, Stony Brook, New York 11794 \\ gwray@life.bio.sunysb.edu
}

Received December 4, 1997.

In writing Cells, Embryos, and Evolution, John Gerhart and Marc Kirschner have tackled a huge, vital, and virtually unexplored topic: how the machinery of cellular processes has influenced the course of morphological evolution. Theirs is a "bottom-up" view of evolution that stresses the opportunism inherent in the way that cells in animal embryos divide, communicate, change shape, and generally go about their business. The point of departure for the book is the observation, initially amazing but now commonplace, that most eukaryotic cells seem to operate using pretty much the same molecular machinery. What is it about these conserved cellular processes that allowed the diversification of metazoans into more than 30 distinct phyla? The authors argue that the answer lies in the "robust flexibility" of animal development: the ability of a core set of cellular processes to direct a much wider range of developmental processes and thereby facilitate the evolution of a broad diversity of phenotypes. The central thesis of this book is that natural selection has produced and maintained a capacity for evolutionary change in animal development that the authors call "evolvability."

For evolutionary biologists looking for an entry into the literature on the evolution of cells and embryos, this book is an informative and thought-provoking introduction. While Cells, Embryos, and Evolution is not without its flaws, the refreshingly original ideas and compendium of interesting examples more than compensate. This review discusses the concept of robust flexibility (a tough cell) and the lack of attention that evolutionary biologists have paid to cellular processes (a tough sell).

\section{Bridging Disciplines and Cultures}

It may initially seem strange that Gerhart and Kirschner have written a book of any importance to evolutionary bi-

\footnotetext{
${ }^{1}$ Cells, Embryos, and Evolution, John Gerhart and Marc Kirschner. 1997. Blackwell Science, Malden, Massachusetts. 642 pp.
PB \$64.95. ISBN 0-86542-574-4.
}

ologists. Although they are distinguished cellular and developmental biologists, neither has published a single peerreviewed paper dealing with evolution. Why did they choose to write a book whose central message is explicitly evolutionary? The answer is simple. It has become abundantly clear that processes within cells and embryos have important evolutionary implications, but for reasons that are not entirely clear, evolutionary biologists have largely ignored these processes. To their great credit, Gerhart and Kirschner have taken some important steps toward placing information about the way cells operate into an evolutionary context.

Interdisciplinary connections are rarely easy to make, and the considerable strengths and weaknesses of this book reflect the attempt to bridge two largely disjunct fields of modern biology. The most important strengths of this book lie in the originality of the ideas and summary of interesting information that it presents. Gerhart and Kirschner are certainly not the first to consider the evolution of developmental processes, but they are among the first to write about the evolutionary implications of cellular processes. Even when discussing the evolution of embryos, a topic that has received much more attention than the evolution of cells, their ideas are often innovative and provocative. Another important strength of the book is its ample and lucid discussion of cell biology for non-specialists. The majority of the 600-plus pages of this book are devoted to extended examples of cellular and developmental processes. This didactic style has the benefit of making the book accessible to all evolutionary biologists. Many of the examples present features of cells that have intriguing but unexplored evolutionary implications, and should encourage evolutionary biologists to begin thinking more about cellular processes. For these reasons, Cells, Embryos, and Evolution deserves to be read by a wide range of evolutionary biologists.

It would be disingenuous, however, to avoid mentioning 